\title{
OPTICALLY DETECTED MAGNETIC RESONANCE STUDIES OF Te-RELATED SHALLOW DONORS IN $\mathrm{Al}_{x} \mathrm{Ga}_{1-x} \mathrm{As}$
}

\author{
M. Godlewski, K. Fronc, \\ Institute of Physics, Polish Academy of Sciences \\ Al. Lotników 32/46, 02-668 Warszawa, Poland
}

W.M. Chen and B. Monemar

Linköping University, Department of Physics and Measurement Technology 58183 Linköping, Sweden

The first studies of the Optically Detected Magnetic Resonance (ODMR) of Te-doped $\mathrm{Al}_{x} \mathrm{Ga}_{1-x} \mathrm{As}(x=0.42)$ are presented. The ODMR data indicate an efficient energy transfer between epilayer and $\mathrm{GaAs}$ substrate.

PACS numbers: 71.55.Eq, 72.20.Jv, 76.70.Hb, 78.55.Cr

\section{Introduction}

Group IV and VI dopants in GaAs form shallow donor states described by the effective mass theory. $A$ remarkably different behaviour is observed once they are introduced to a ternary $\mathrm{Al}_{x} \mathrm{Ga}_{1-x}$ As. For $x>0.2$ a deep donor state is created, commonly called the $\mathrm{DX}$ center [1], accompanied by a metastable effective mass-like state. The ODMR was recently applied to the studies of recombination processes in Si-, S- and Se-doped AlGaAs [2, 3]. We present here the first ODMR studies of Te-doped AlGaAs.

\section{Experimental}

The ODMR studies were performed on a converted ESR spectrometer Bruker 200 D-SRC, equipped with an Oxford Instruments ESR 10 continuous liquid $\mathrm{He}$ flow cryostat. PL was excited with the $514.5 \mathrm{~nm}$ line of an $\mathrm{Ar}^{+}$laser and detected 


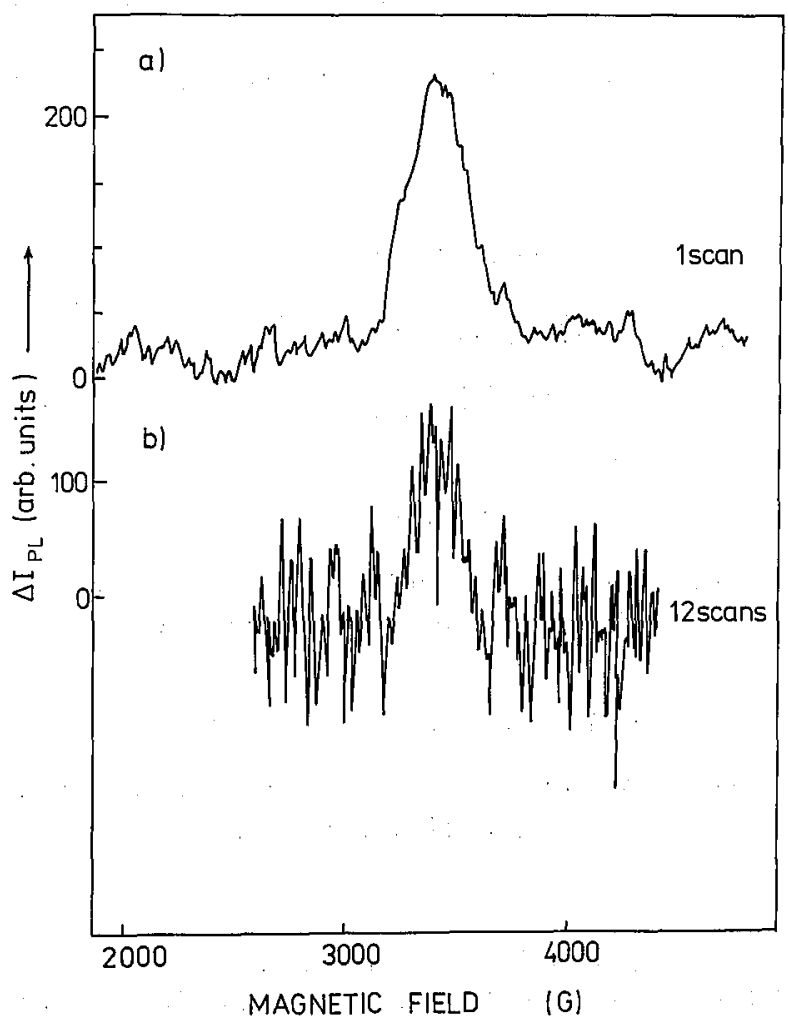

Fig. 1. Magnetic resonance signal of the AlGaAs epilayer under the band gap excitation. The signal was detected at $12 \mathrm{kHz}$ microwave chopping frequency via the change in intensity of "shallow" C-related GaAs (a) and AlGaAs (b) DAP emissions.

via a $0.25 \mathrm{~m}$ Jobin-Yvon monochromator with a cooled North Coast EO $817 \mathrm{Ge}$ detector and S-1 or S-20 photomultipliers.

The AlGaAs layers were grown by Liquid Phase Epitaxy on a semi-insulating [001] GaAs:Cr substrate. Relatively thick epilayers were used in the experiment (about $45 \mu \mathrm{m}$ ). They were doped with Te to a $10^{16} \mathrm{~cm}^{-3}$ level. The Al fraction was about 0.42 .

\section{Experimental results and discussion}

In Fig. 1(a) we show the ODMR signal observed via the C-related DAP emission of GaAs. This signal was observed only if the AlGaAs layer was excited directly. The spectral dependence of this signal is shown in Fig. 2(b). Signal averaging was necessary to prove that it originates also from the AlGaAs DAP emission at $1.988 \mathrm{eV}$ (Fig. 1(b)). The ODMR signal was observed as an enhancement of both a direct radiative recombination and an energy transfer to GaAs. The data 


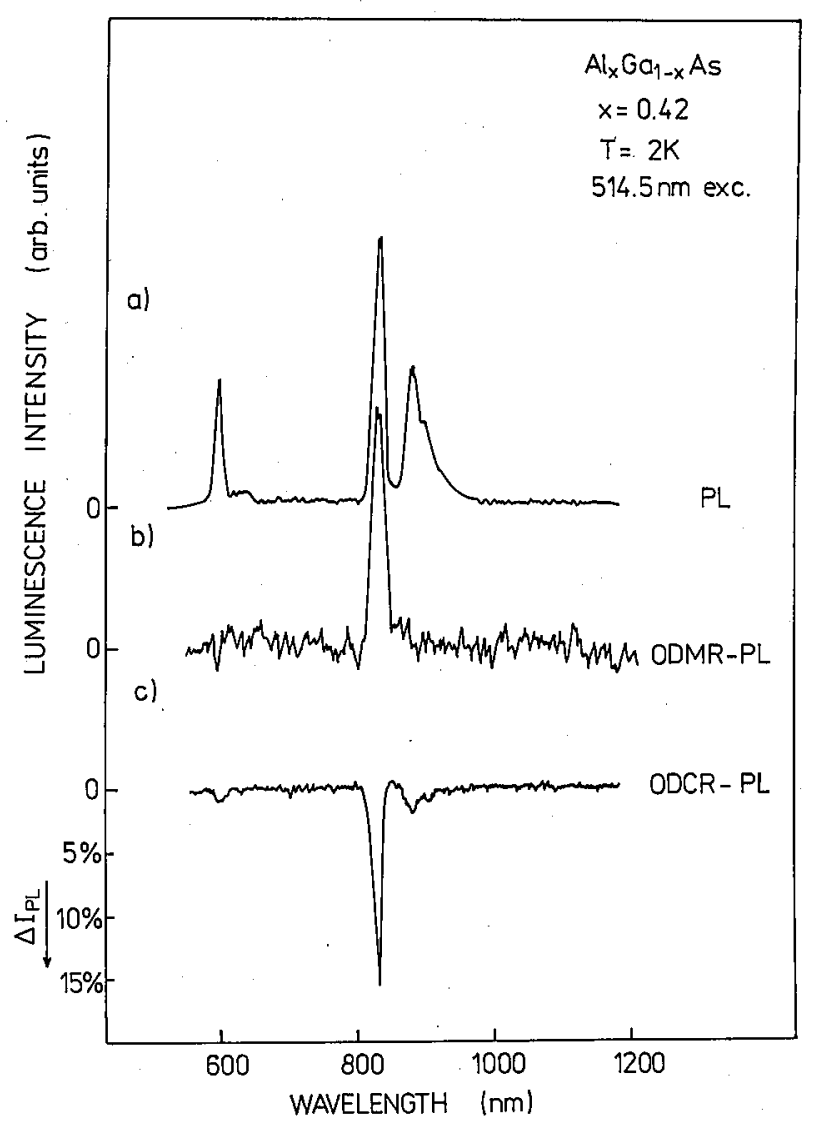

Fig. 2. PL spectrum of $\mathrm{AlGaAs}$ epilayer and GaAs substrate measured under the $514.5 \mathrm{~nm}$ excitation of AlGaAs (a). The relative intensities of the emissions are affected by the response of the detector. The spectrum consists of AlGaAs bound exciton $(2.06 \mathrm{eV})$ and DAP $(1.988 \mathrm{eV})$ emissions, and of two GaAs DAP transitions at $1.488 \mathrm{eV}$ and $1.4 \mathrm{eV}$. In (b) the spectral dependence of the donor signal is shown. The data shown in (c) present the reduction of PL spectra due to impact ionization of excitons and shallow donors by the microwave heated free carriers.

shown in Fig. 2(b) indicate, thus, an efficient transfer link between epilayer and substrate. There are two possible mechanisms which can explain the above observation. Such a link is either due to a free carrier migration from the epilayer or/and. due to an energy transfer from AlGaAs to GaAs [4]. The ODMR (positive sign of the resonance signal from both AlGaAs and GaAs DAP emissions) and impact ionization studies [5] (Fig. 2(c)) indicate that the last mechanism dominates.

The obvious question is the nature of the resonance signal observed. Since it arises from the AlGaAs DAP transition it can be attributed to either a donor or an acceptor resonance. A shallow acceptor signal is difficult to observe due to 
an unlifted orbital degeneracy. We will, thus, present here arguments that the observed signal is due to a metastable $X$-related "shallow" donor in AlGaAs. The signal has a $g$-factor about 1.95 . Such a $g$-factor is inconsistent with the one expected for $\Gamma$ minimum shallow donors [6]. A typical $g$-value for the indirect band gap Ge with a $L$ conduction band (CB) minimum is about 1.5 [7]. For indirect band gap $\mathrm{GaP}$ with a lowest $X \mathrm{CB}$ minimum $g$-factors about 2 were observed for group VI donors [8]. The resonance signal observed can, thus, be attributed to a "X-like" shallow donor in AlGaAs.

In the effective mass approximation the $X$ CB minimum-related group VI donors should have an $A_{1}$ symmetry ground state [9]. The magnetic resonance signal should, thus, be isotropic. In contrary anisotropic signals were observed for $S$ and Se [3] and in our studies for Te. This anisotropy is heteroepitaxial strain-related. A tensile strain along the epilayer growth axis ([001]) is introduced due to a smaller lattice constant of GaAs. The observed anisotropy is due to a strain-induced mixing of an excited $E$ symmetry state and reflects an anisotropy of this state and a magnitude of the valley-orbit splitting [3]. Due to the large width of the ODMR signal we could not determine this anisotropy accurately.

Concluding, all the features of the resonance signal are consistent with its $X$ CB "metastable" shallow donor origin. We have not observed any signal or a radiative transition which could be directly related to the deep DX center.

\section{References}

[1] D.V. Lang, R.A. Logan, M. Jaros, Phys. Rev. B 19, 1015 (1979).

[2] T.A. Kennedy, E. Glaser, Solid State Phenomena 10, 53 (1989).

[3] E. Glaser, T.A. Kennedy, B. Molnar, M. Mizuta, Mat. Res. Soc. Symp. Proc. 163, 753 (1990).

[4] R.J. Roedel, V.G. Karamandas, J. Appl. Phys. 50, 6353 (1979).

[5] H. Weman, M. Godlewski, B. Monemar, Phys. Rev. B 38, 12525 (1988).

[6] H.J. von Bardeleben, Solid State Phenomena 10, 181 (1989).

[7] G. Feher, D.K. Wilson, E.A. Gere, Phys. Rev. Lett. 3, 25 (1959).

[8] F. Mehran, T.N. Morgan, R.S. Title, S.E. Blum, Phys. Rev. B 6, 3917 (1972).

[9] T.N. Morgan, Phys. Rev. B 34, 2664 (1986). 